\title{
Fine Needle Aspiration Cytology of Liver Space Occupying Lesions - A Study of 57 Cases from a Tertiary Care Centre
}

\author{
Neeraj Dhameja ${ }^{1}$, Varnika Rai ${ }^{2}$, Rajeev Singh $^{3}$ \\ ${ }^{1}$ Assiatant Professor, Department of Pathology, Institute of Medical Sciences, Banaras Hindu University, Varanasi-India \\ ${ }^{2}$ Junior Resident-3, Department of Pathology, Institute of Medical Sciences, Banaras Hindu University, Varanasi-India \\ ${ }^{3}$ Senior Resident, Department of Radiodiagnosis and Imaging, Institute of Medical Sciences, Banaras Hindu University, Varanasi-India
}

\begin{abstract}
Ultrasound guided fine needle aspiration cytology helps in diagnostic evaluation of hepatic lesions. It provides a predictive diagnosis of benign or malignant neoplasm and in many cases also of specific tumor type. In our study we are describing cytological features of liver SOL in 57 cases who came to SS hospital BHU Varanasi within two years duration. Age range was 2 years to 70 years. Primarily the diagnosis was made on conventional cytological stains (Giemsa and Papanicolaou stain stain). Of the total 57 cases, 54 cases (94.7\%) were diagnosed as neoplastic and three cases as non-neoplastic lesions (5.2\%). Of total 54 cases 12 neoplastic lesions (22.2\%) were diagnosed as primary hepatic malignancies in which four (7.4\%) were Hepatoblastomas and eight (14.8\%) Hepatocellular carcinomas, of which six were adult HCCs and two were pediatric HCCs. Secondary (metastatic) hepatic malignancies comprised 42 cases of total 54 neoplastic lesions (77.7\%), 34 Adenocarcinomas (63\%), 2 squamous cell carcinomas (SCC) (3.7\%), 2 neuroendocrine tumours (3.7\%), 2 melanomas (3.7\%), one Non Hodgkin Lymphoma (NHL) (1.8\%) and one Anaplastic carcinoma (1.8\%). The three non-neoplastic lesions (5.3\%) included two granulomatous lesions with necrosis and one hydatid cyst. FNAC is a safe and rapid diagnostic tool for hepatic masses.
\end{abstract}

Keywords: Liver SOL, Hepatocellular carcinoma, Hepatoblastoma, Metastatic Adenocarcinoma, Primary malignancy.

\section{Introduction}

Liver can be affected by a variety of diseases which can be metabolic, infectious or neoplastic and present radiologically either as diffuse involvement or as focal lesion also designated as space occupying lesions (SOLs). These diseases are suspected clinically, biochemically and radiologically, however, tissue from the lesion is often required for definite diagnosis. Fine needle aspiration cytology (FNAC) is a rapid, inexpensive and minimally invasive technique for diagnosis of liver space occupying lesions without significant complications. ${ }^{[1]}$ Radiologically guided FNAC [either ultrasound (US) or computerized tomography (CT) guided] is commonly done as multiple passes can be done from different representative sites and cell blocks can be made for immunohistochemistry and molecular testing. The diagnostic accuracy of FNAC for liver lesions is greater than $85 \%{ }^{[2]}$ and in malignant lesions its sensitivity is around $90 \%(67-100 \%) .{ }^{[1]}$

The aim of this study was to evaluate the different hepatic lesions diagnosed on FNAC presenting in our hospital.

\section{Materials and Methods}

A total of 57 cases of liver lesions who presented to SS Hospital, IMS, BHU during a two-year period (2014-2015) and where FNA yielded adequate cellularity were selected for this study. The patients age ranged from 2 to 70 years and patients presented with either pain right upper quadrant with abdominal mass or fatigue and radiologically detected liver SOLs. The coagulation profile was normal in all patients. In all cases US guided FNAC was done using 21-23 G needle. Air dried and alcohol fixed smears were made and stained with Giemsa and Papaniocoloau stains. No complication was observed in any case after FNAC.

\section{Observation and Result}

The patients age ranged from 2 to 70 years. Male to female ratio is 4:1. Table 1 shows number of cases of different cytological diagnoses and respective percentages.

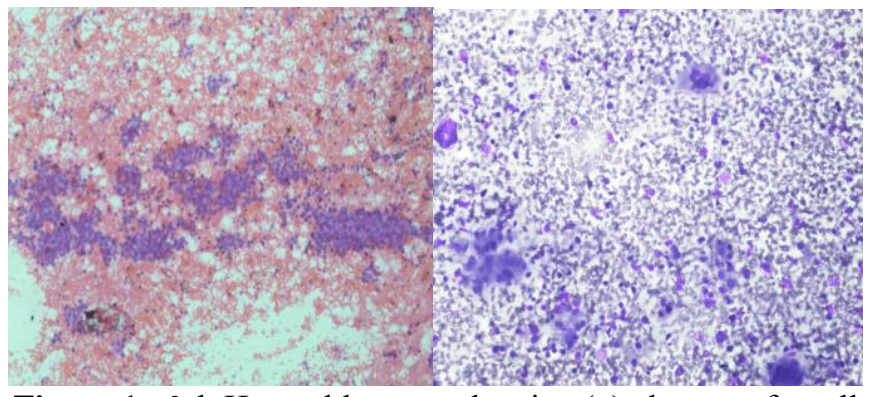

Figure $1 \mathrm{a} \& \mathrm{~b}$ Hepatoblastoma showing (a) clusters of small round cells with a small amount of cytoplasm (PAP, X100) and (b) extramedullary hematopoiesis as megakaryocyte seen. (Giemsa, X400) 


\section{International Journal of Science and Research (IJSR) \\ ISSN (Online): 2319-7064}

Index Copernicus Value (2013): 6.14 | Impact Factor (2014): 5.611

Table1: spectrum of FNAC findings liver SOL

\begin{tabular}{|c|c|c|c|}
\hline & Diagnosis on FNAC & $\begin{array}{c}\text { No of } \\
\text { cases }\end{array}$ & $\begin{array}{c}\text { Percentage } \\
(\%)\end{array}$ \\
\hline A. & Neoplastic lesion & $\underline{\mathbf{5 4}}$ & $\mathbf{9 4 . 7}$ \\
\hline $\mathbf{1 .}$ & Primary malignancy & $\mathbf{1 2}$ & $\mathbf{2 1 . 0}$ \\
\hline a & Hepatoblastoma (fig 1a \& b) & 4 & 7.0 \\
\hline b & Pediatric hepatocellular carcinoma & 2 & 3.5 \\
\hline c & Adult HCC (fig 2a \& 2b) & 6 & 10.5 \\
\hline $\mathbf{2 .}$ & Secondary malignancy & $\mathbf{4 2}$ & $\mathbf{7 3 . 7}$ \\
\hline a. & Adenocarcinoma (fig 3a and b) & 34 & 59.6 \\
\hline b & Squamous cell carcinoma (fig 4) & 2 & 3.5 \\
\hline c. & Neuroendocrine tumour (fig 5) & 2 & 3.5 \\
\hline d. & Melanoma (fig 6) & 2 & 3.5 \\
\hline e. & Non Hodgkin's lymphoma (fig 7) & 1 & 1.8 \\
\hline f & Anaplastic carcinoma & 1 & 1.8 \\
\hline B. & Non neoplastic disease & $\underline{\mathbf{3}}$ & $\underline{\mathbf{5 . 3}}$ \\
\hline a. & Granuloma (fig. 9) & 2 & 3.5 \\
\hline b. & Hydatid cyst (fig 10) & 1 & 1.8 \\
\hline
\end{tabular}

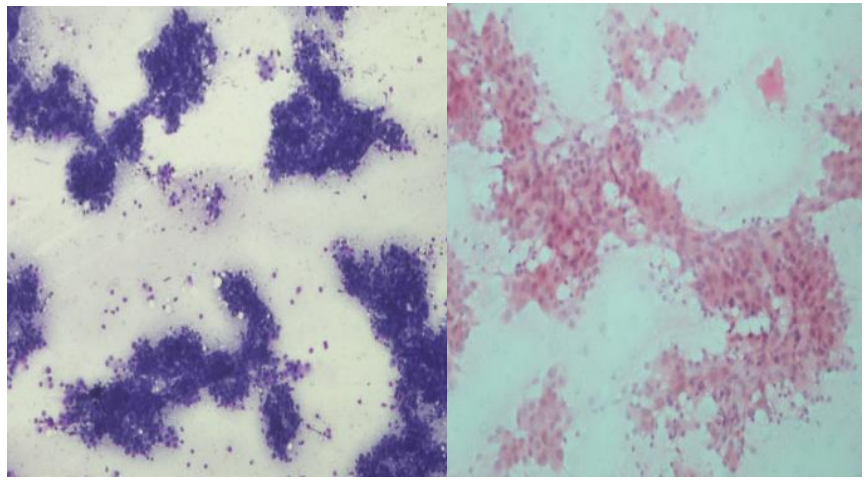

Figure 2: a Hepatocellular carcinoma with abnormally thick trabecular cords and bare nuclei (Giemsa, X 100). Fig 2b

Polygonal hepatocytes show increased nucleus-tocytoplasmic $(\mathrm{N}: \mathrm{C})$ ratio, rounded nuclei, abundant granular eosinophilic cytoplasm and intracytoplasmic bile; cluster of malignant cells traversed by spindle endothelial cells (PAP, $\times 400)$.

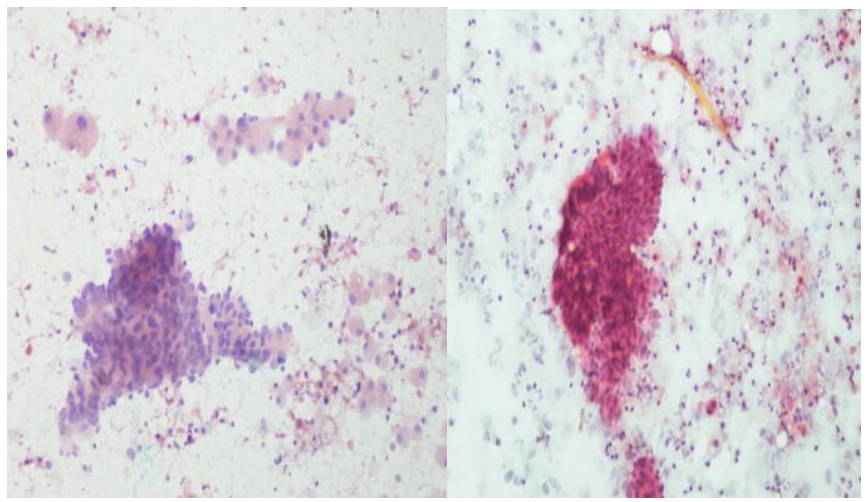

Figure 3(a). Colon carcinoma metastatic to the liver, showing glandular arrangement of cells with background of substantial necrosis and cluster of hepatocyte (PAP, X200). (b). Metastatic colon carcinoma with cigar-shaped nuclei. (PAP, X200)

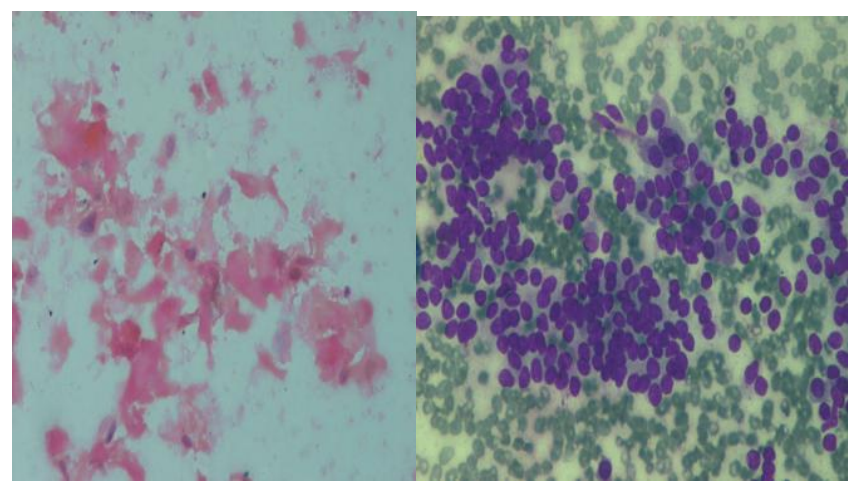

Figure 4: Metastatic squamous cell carcinoma with hyperchromatic nuclei and abundant eosinophilic cytoplasm,

(PAP X 400). Figure 5: Low grade neuroendocrine carcinoma, showing rosette-like aggregates and eccentric nuclei. No necrosis or mitoses present.

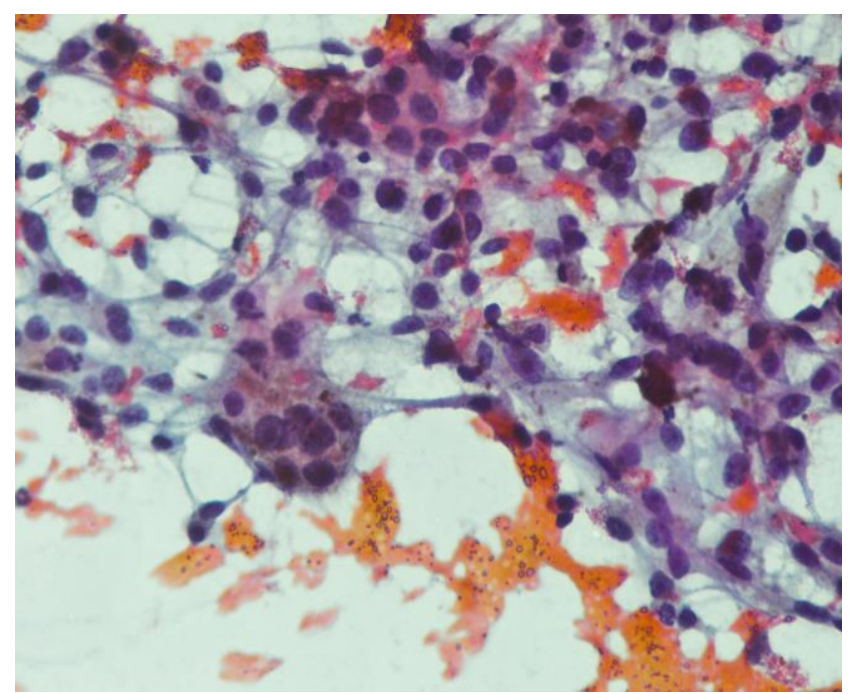

Figure 6: Melanoma metastatic to liver with copious melanin pigment and pleomorphic hyperchromatic nuclei,(PAP, $\mathrm{X} 400$ )

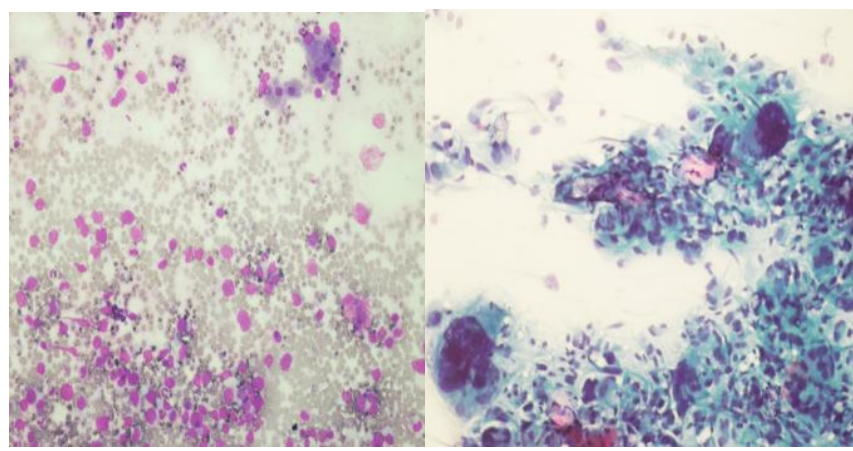

Figure 7: Non-Hodgkin's lymphoma: Fine-needle aspiration cytology: Dispersed large lymphoid cells showing pale nuclei, and scanty cytoplasm (Giemsa, X400). Figure 8: Anaplastic ca pancreas metastasis with highly pleomorphic nuclei, giant cell formation in a necrotic background. (PAP, $\mathrm{X} 400)$ 


\section{International Journal of Science and Research (IJSR) \\ ISSN (Online): 2319-7064}

Index Copernicus Value (2013): 6.14 | Impact Factor (2014): 5.611

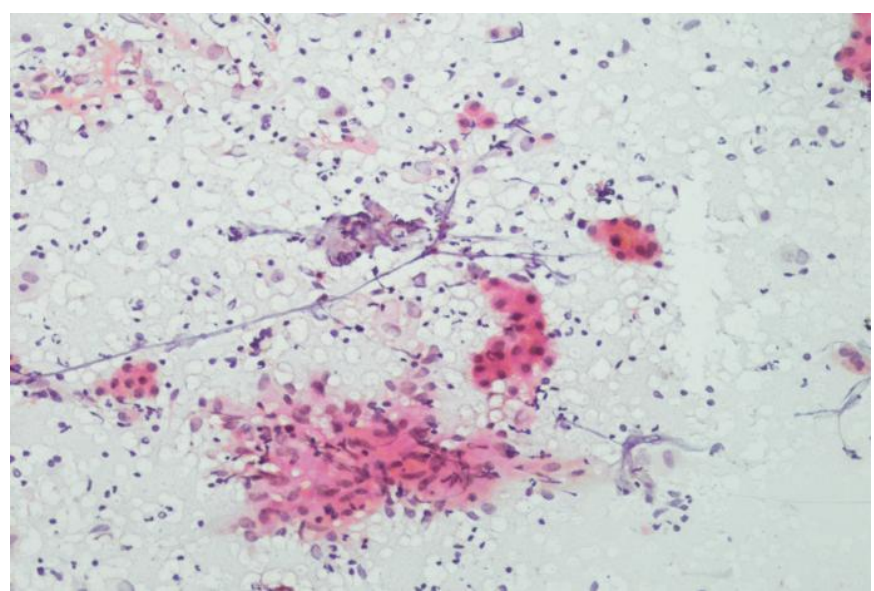

Figure 9: Epitheloid cell granuloma with hepatocyte clusters in background. (20 X, PAP)

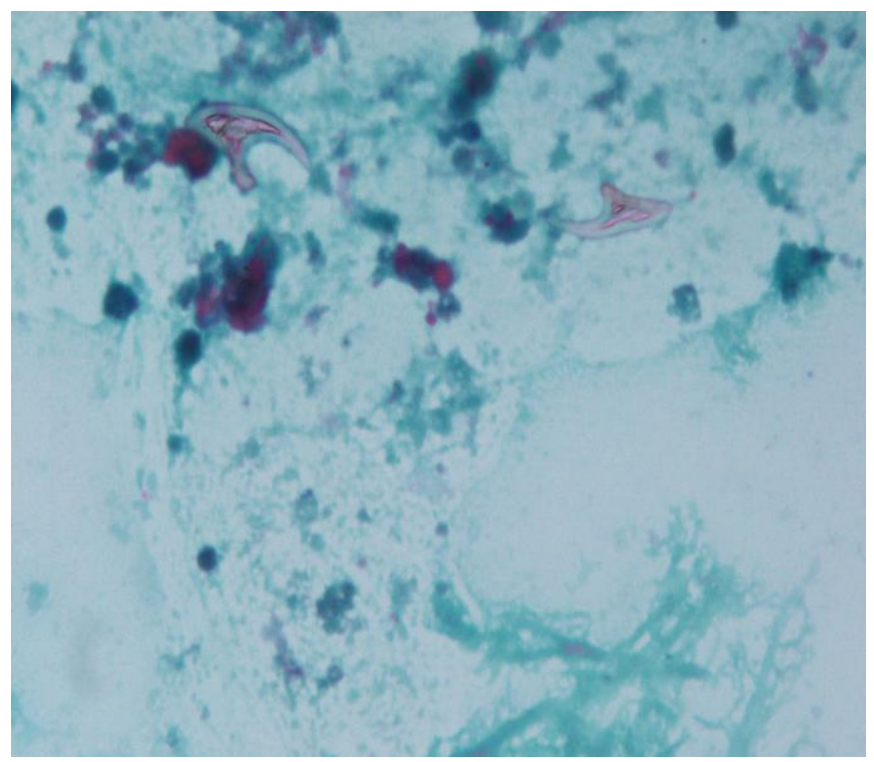

Figure 10: Hydatid cyst FNAC: Refractile hooklets with adjacent inflammatory and degenerated cells (PAP, X1000).

\section{Discussion}

FNAC is an established diagnostic modality to diagnose hepatic space occupying lesions and helps to categorize them into neoplastic or nonneoplastic lesions. Lundquist et al in $1971^{[2]}$ first showed the utility of FNAC in diagnosing hepatic lesions. Various subsequent studies on fine needle aspiration cytology of hepatic lesions have been done and their results have confirmed that it is a very useful modality to diagnose hepatic lesions. ${ }^{[3-8]}$ A study by Das et al of 212 liver SOLs found 93 malignant lesions with accurate classification into primary and secondary types and concluded that guided FNAC plays an important role in diagnosis and classification of liver malignancies. ${ }^{[9]}$ Multiple passes from different sites can be performed and cell blocks can be made which can be utilized for immunohistochemistry and molecular studies. No significant complications are seen and the only contraindications are bleeding diathesis and vascular lesions. ${ }^{[1]}$ In this study, 57 liver US guided FNACs were included with adequate cellularity. The patients included 4 children (age range 2-10 years) and 53 adults (age range 2570 ). The clinical presentation was pain and mass abdomen and fatigue. Ultrasound examination showed solitary and multiple hepatic space occupying lesions. Neoplastic and non neoplastic lesions were 54 and 3 in number and Primary and secondary (metastasis) hepatic malignancies represented $21.0 \%$ and $73.7 \%$ respectively. Other studies also have shown that metastatic lesions are more common than primary hepatic malignancies ${ }^{[5,7,8]}$, however, study by Swamy et al ${ }^{[6]}$ found primary malignancies more common than metastatic lesions. Primary hepatic malignancies in this study included Hepatoblastoma (4 cases) and Hepatocellular carcinoma (6 adults and 2 pediatric HCCs). All hepatoblastoma cases showed epithelial elements with foci of extramedullary haematopoeisis. One case of hepatoblastoma was included in the study by Gayatri et al ${ }^{[5]}$, however, pediatric HCCs have not been described in other studies. One patient of adult HCC had history of alcohol intake and one was a known case of hepatitis $\mathrm{C}$ virus infection. Ultrasound examination in both these patients showed background cirrhotic changes with SOLs. The diagnosis of HCC was made on architectural and cytological features as described in standard cytology textbooks and many studies. ${ }^{[1,2,10-13]}$ The architectural features included trabecular pattern with wrapping or transgressing endothelial cells and bare atypical nuclei in the background. The cytological features included polygonal cells with centrally placed nuclei with high nucleocytoplasmic ratio, prominent nucleoli and moderate eosinophilic cytoplasm. Metastatic malignancies were more common in this study. Adenocarcinoma was the most common (34 in number). Squamous cell carcinoma (SCC), malignant melanoma and neuroendocrine tumour represented two cases of each and one case of NHL and one case of Anaplastic carcinoma. Adenocarcarcinoma was diagnosed based on three dimensional clusters and gland formation by cuboidal to columnar malignant cells with benign hepatocytes. Gastrointestinal tract was the most common site and one patient was a follow up case of carcinoma breast. One patient each of metastatic SCC and malignant melanoma were known cases of primary cervical carcinoma and vulval melanoma respectively. One case of NHL, large cell type was included in this study. No history of primary lesion elsewhere or lymphadenopathy was present. A study by Gayatri et $\mathrm{al}^{[5]}$ also included 4 cases of large cell type of NHL. Two metastatic neuroendocrine tumours were included in this study. One patient had carcinoid syndrome with flushing and diarrhea and other patient had history of surgery for mass in the small intestine one year back, however, no records were available. The patient with metastatic anaplastic carcinoma had primary in the pancreas.

Three non-neoplastic lesions were included in this study and represented 2 cases of granulomatous lesions with necrosis and one case of hydatid cyst. Stain for acid fast bacilli was done which was noncontributory. Patient with granulomatous lesions were started on antitubercular therapy and responded. A study by Radhika et al ${ }^{[14]}$ showed 4 cases of hepatic tuberculosis diagnosed by FNAC. Smears from the patient with hydatid cyst showed refractile hooklets which were confirmed histopathologically. No complication was seen after FNAC.

Benign hepatic neoplastic lesions were not seen in this study.

Hence, this study shows different neoplastic and non-

\section{Volume 5 Issue 2, February 2016}


neoplastic lesion can be accurately diagnosed by guided FNAC as shown by other studies.

\section{Conclusion}

FNAC is a safe and rapid diagnostic tool for hepatic masses and can segregate benign and malignant lesions and primary and secondary malignancies with accuracy.

\section{References}

[1] Boer BD. Liver and Spleen. In: Orell SR and Sterrett GF, editors. Fine Needle Aspiration Cytology. $5^{\text {th }}$ ed. New Delhi:Elsevier;2012.271-96.

[2] Pitman MB. Liver. In: Gray W, Kocjan G, editors. DiagnosticCytopathology. $3^{\text {rd }}$ ed.Philadelphia:Elsevir;201 0. 287-317.

[3] Pinto MM, Avila NA, Heller CI, Criscuolo EM. Fine Needle Aspiration of the liver. Acta Cytol.1988;32:1521.

[4] Shah A, Jain GM. Fine needle aspiration cytology of Liver- A study of 518 cases. J Cytol.2002;19:139-43.

[5] Gatphoh ED, Gaytri S, Babina S, Singh AM. Fine needle aspiration cytology of liver - a study of 202 cases. Indian Journal of Medical Sciences.2003;57:22-25.

[6] Swamy MCM, Arathi CA, Kodandaswamy CR. Value of ultrasonography guided fine needle aspiration cytology in the investigative sequence of hepatic lesions with an emphasis on hepatocellular carcinoma. J Cytol.2011;28:178-84.

[7] Rasania A, Pandey CL, Joshi N. Evaluation of FNAC in Diagnosis of Hepatic Lesion. J Cytol. 2007;24(1):51-54.

[8] Ali SR, Jayabackthan L, Rahim S, Sharel MB, Prasad K, Hegdekatte N. Role of fine needle aspiration cytology in the diagnosis of hepatic lesions. Muller Journal of Medical Sciences and Research. 2015;6:125-28.

[9] Das DK, Tripathi RP, Kumar N, et al. Role of guided fine needle aspiration cytology in diagnosis and classification of liver malignancies. Trop Gastroenterol. 1997; 18:101-6.

[10]Bottles K, Cohen MB, Holley EA, et al. A Step- Wise Logistic Regression Analysis of Hepatocellular Carcinoma. An Aspiration Biopsy Study. Cancer.1988;62:558-63.

[11]Cohen MB, Haber MM, Holley EA, Ahm DK, Bottus K, Sitoloff AC. Cytologic criteria to distinguish hepatocellular carcinoma from nonneoplastic liver. Am J Clin Pathol.1991;95:125-30.

[12]Devi VL, Hazarika D. Fine Needle Aspiration Cytology of Hepatocellular Carcinoma. Indian $\mathrm{J}$ Pathol Microbiol.1995;38:389-92.

[13] Ahuja A, Gupta N, Srinivasan R, Chawla Y, Rajwanshi A. Differentiation of Hepatocellular Carcinoma from Metastatic Carcinoma of the Liver - Clinical and Cytological Features. J Cytol.2007;24(3):125-29.

[14] Radhika S, Rajwanshi A, Kochhar R, Kochhar S, Dey P, Roy P. Abdominal tuberculosis. Diagnosis by fine needle aspiration cytology. Acta Cytol.1993;37:673-78. 